\title{
Genomic Signature of MTOR Could Be an Immunogenicity Marker in Human Colorectal Cancer
}

\section{Chenxing Wang}

Shanghai Jiao Tong University Medical School Affiliated Ruijin Hospital

\section{Batuer Aikemu}

Shanghai Jiao Tong University Medical School Affiliated Ruijin Hospital

\section{Yanfei Shao}

Shanghai Jiao Tong University Medical School Affiliated Ruijin Hospital

\section{Sen Zhang}

Shanghai Jiao Tong University Medical School Affiliated Ruijin Hospital

\section{Guang Yang}

Shanghai Jiao Tong University Medical School Affiliated Ruijin Hospital

\section{Hiju Hong}

Shanghai Jiao Tong University Medical School Affiliated Ruijin Hospital

\section{Ling Huang}

Shanghai Jiao Tong University Medical School Affiliated Ruijin Hospital

\section{Hongtao Jia}

Shanghai Jiao Tong University Medical School Affiliated Ruijin Hospital

\section{Xiao Yang}

Shanghai Jiao Tong University Medical School Affiliated Ruijin Hospital

\section{Minhua Zheng}

Shanghai Jiao Tong University Medical School Affiliated Ruijin Hospital Jing Sun ( $\nabla$ sj11788@rjh.com.cn )

Shanghai Jiao Tong University Medical School Affiliated Ruijin Hospital https://orcid.org/0000-00032110-5189

\section{Jianwen Li}

Shanghai Jiao Tong University Medical School Affiliated Ruijin Hospital

\section{Research Article}

Keywords: MTOR, colorectal cancer, immune infiltrates, microsatellite instability, whole-exome sequencing Posted Date: February 21st, 2022 
DOI: https://doi.org/10.21203/rs.3.rs-1249776/v1

License: (c) (1) This work is licensed under a Creative Commons Attribution 4.0 International License. Read Full License 


\section{Abstract \\ Background}

The mTOR signaling pathway plays an important role in cancer. As a master regulator, the status of MTOR affects pathway activity and the efficacy of mTOR inhibitor therapy. However, little research has been performed to explore MTOR in colorectal cancer (CRC).

\section{Methods}

In this study, gene expression and clinical data were analyzed using The Cancer Genome Atlas (TCGA) and the Gene Expression Omnibus (GEO) databases. Signaling pathways related to MTOR in CRC were identified by Gene Ontology (GO), Kyoto Encyclopedia of Genes and Genomes (KEGG), and gene set enrichment analysis (GSEA). Somatic mutation data were downloaded from TCGA and analyzed using the maftools R package. Tumor Immune Estimation Resource (TIMER) and CIBERSORT were used to analyze correlations between MTOR and tumor-infiltrating immune cells (TIICs). Finally, we detected MTOR mutations in a CRC cohort from our own database using whole-exome sequencing.

\section{Results}

We found that MTOR was overexpressed in Asian CRC patients and associated with a poor prognosis. Enrichment analysis showed that MTOR was involved in metabolism, cell adhesion, and translation pathways in CRC. High MTOR expression was correlated with high tumor mutation burden (TMB) and several TIICs. Finally, we found that the mTOR signaling pathway was activated in CRC lines characterized by microsatellite instability (MSI), and the frequency of MTOR mutations was higher in MSI-high (MSI-H) patients than in microsatellite stable (MSS) patients.

\section{Conclusions}

MTOR may represent a comprehensive indicator of prognosis and immunological status in CRC. The genomic signatures of MTOR may provide guidance for exploring the role of mTOR inhibitors in CRC.

\section{Introduction}

Mammalian target of rapamycin (mTOR), the protein product of the MTOR gene, is a serine/threonine kinase and an important downstream effector of the phosphoinositide 3-kinase (PI3K)/protein kinase B (AKT) pathway. In mammalian cells, the PI3K/AKT/mTOR pathway plays a crucial role in the regulation of cell survival, metabolism, growth, and protein synthesis in response to upstream signals during both normal physiological and abnormal pathological conditions (1). This pathway represents one of the most deregulated signaling pathways in human cancer. mTOR is considered to serve as a master regulator of 
this signaling pathway, and recent findings have reported that mTOR activation plays a vital role in human cancer (2). As reported, mTOR is aberrantly overactivated in more than $70 \%$ of cancers (3). In lung cancer, the activation of the mTOR pathway is associated with poor prognosis $(4,5)$. In breast cancer, mTOR expression correlated with worse prognosis, and p-mTOR was more commonly detected in triplenegative breast cancers $(6,7)$. The abnormality of mTOR activation in cancer offers opportunities for targeted therapy. Several distinct classes of drugs have been developed to inhibit mTOR, including antibiotic allosteric mTOR inhibitors (rapamycin and its rapalogs), ATP-competitive mTOR inhibitors, and mTOR/PI3K dual inhibitors. Several mTOR inhibitors have reached various stages of clinical trials, but only temsirolimus and everolimus have been approved by the Food and Drug Administration for clinical use in the treatment of cancer patients. Preclinical studies have demonstrated the efficacy of most mTOR inhibitors in several types of cancers; however, early clinical trials have yielded mixed results because inhibitors (such as ATP-competitive mTOR inhibitors and mTOR/PI3K dual inhibitors) were often associated with dose-limiting toxicities, possibly due to low selectivity (8-12). The degree of dependence on mTOR activation varies, and even among patients with similar mutation profiles, the outcomes associated with mTOR inhibitor therapy have been reported to vary across different cancer types. Although temsirolimus has been approved for the treatment of patients with advanced-stage renal cell carcinoma (RCC), clinical experience has indicated that only $8.6 \%$ of patients experienced an objective response in the phase III Global advanced-stage RCC trial (13). Identifying predictive biomarkers capable of determining which patients might benefit from mTOR inhibitors remains necessary. Mutations in MTOR have been shown to alter the sensitivity to rapalog treatment in several cancer types (14-16). Therefore, the molecular alterations of MTOR warrant further investigation.

Colorectal cancer (CRC) is the second most common cause of cancer-related deaths worldwide (17) and represents one of the most common malignant tumors in China. The incidence and mortality of CRC in China ranked third and fifth, respectively, among all malignant tumors in 2015 , associated with 388,000 new cases and 187,000 deaths (18). Although developments in targeted therapy and immunotherapy have improved outcomes for some patients, the prognosis of CRC remains far from satisfactory for the majority of patients (19). Therefore, the identification of potential prognostic biomarkers and novel treatment targets for $\mathrm{CRC}$ remains necessary. Several studies have examined the role of the $\mathrm{PI} 3 \mathrm{~K} / \mathrm{AKT} / \mathrm{mTOR}$ pathway in CRC, but most of these have focused on upstream regulators, such as phosphatase and tensin homolog (PTEN) and phosphatidylinositol-4,5-bisphosphate 3-kinase catalytic subunit alpha (PIK3CA), with few studies focused on mTOR itself. Previous studies (20-23) have demonstrated that mTOR is highly activated in CRC, associated with the proliferation of CRC. However, the functional outcomes and the underlying activating mechanisms of MTOR in CRC remain to be investigated. Currently, the efficacy of mTOR inhibitors is limited in CRC, which may be related to the lack of study regarding MTOR function in this cancer type.

In this study, MTOR expression in CRC and the correlations between MTOR expression and prognosis were analyzed using datasets obtained from The Cancer Genome Atlas (TCGA) and Gene Expression Omnibus (GEO) databases. Differential gene expression analyses, including Gene Ontology (GO), Kyoto Encyclopedia of Genes and Genomes (KEGG) pathway enrichment analysis, and gene set enrichment 
analysis (GSEA), were used to investigate the possible molecular functions of MTOR in CRC. We also analyzed the correlation between MTOR and tumor mutational burden (TMB), the tumor immune microenvironment, and microsatellite instability (MSI) status. Finally, for further exploration and verification, we examined the single-nucleotide variations in the MTOR sequence among an Asian population cohort from our own database. Our findings indicate the prognostic value of MTOR in CRC and demonstrate the potential associations between MTOR and tumor mutation. We hope this study will contribute to new prognostic monitoring and treatment strategies for CRC patients.

\section{Materials And Methods}

\subsection{Data acquisition and gene expressional analysis}

The mRNA levels of MTOR in cancer and para-cancer tissues were analyzed through the "Differential Expression" module in Tumor Immune Estimation Resource (TIMER;

https://cistrome.shinyapps.io/timer/)(24). Gene expression data and corresponding clinical data for the CRC samples included in TCGA datasets (colon carcinoma [COAD] and rectal carcinoma [READ]) were obtained from the University of California Santa Cruz (UCSC, https://xenabrowser.net/datapages/). Corresponding somatic mutation data were obtained from the TCGA website (https://portal.gdc.cancer.gov/)(25). In addition, we selected four datasets (GSE41657, GSE113513, GSE87211, and GSE75316) from the GEO database. The GSE41657 and GSE113513 datasets contain both CRC tissue and normal colon tissue samples from Asian patients. The GSE87211 dataset was used to perform a prognostic analysis of CRC patients. The GSE75316 dataset is comprised of microsatellite stable (MSS), MSI-high (MSI-H), and MSI-low (MSI-L) CRC patients.

\subsection{Overall survival analysis}

Survival analyses in the TCGA and GSE87211 datasets were compared between high and low MTOR expression groups based on cutoff levels established at the median value (50\%) and the quantile values (the top 25\% and the bottom 25\%) of MTOR expression. Kaplan-Meier curves were generated using the "survival" R package.

\subsection{Differentially expressed genes and functional enrichment analysis}

Differentially expressed genes (DEGs) between the high and low MTOR expression groups (determined by quantile) in the TCGA dataset were identified using the limma $\mathrm{R}$ package with the parameters of $\log _{2}$ fold change $\left(\log _{2} F C\right) \mid>0.5$ and false discovery rate $(F D R)<0.05$. For further functional enrichment analysis of DEGs, GO enrichment analysis, including cellular components (CC), molecular functions (MF), and biological processes (BP), and KEGG analysis were performed using the clusterProfiler R package and visualized by the OmicShare tools, a free online platform for data analysis (http://www.omicshare.com/tools). GSEA software (https://www.gsea-msigdb.org/gsea/login.jsp/) was also utilized to analyze the enriched pathways of identified DEGs. 


\subsection{Mutation analysis in CRC}

The maftools R package (26) was used to analyze and visualize the original MAF files of all CRC patients and some of the CRC patients from the high and low MTOR expression groups in TCGA. The maftools R package was also applied to visualize the mutation signatures of genes associated with the mTOR pathway. TMB was calculated as the number of somatic nonsynonymous variations in the TCGA datasets. The "Mafcompare" function in the maftools R package was utilized to identify differentially mutated genes between high and low MTOR expression groups.

\subsection{Characteristic of the tumor microenvironment}

The "Gene" and "SCNA" modules of TIMER were applied to explore the correlations between MTOR and the abundance of six subtypes of immune cell infiltrates (B cells, CD $4^{+} T$ cells, CD $8^{+} T$ cells, neutrophils, macrophages, and dendritic cells) in CRC. Considering the important role of immune cells in the tumor microenvironment (TME), the CIBERSORT method (https://cibersort.stanford.edu/)(27) was used to further quantify the proportions of 22 immune infiltration cells in CRC samples between the high and low MTOR expression groups.

\subsection{CRC lines and Cell culture}

HCT-116, RKO, SW-620, and HT-29 cell lines were obtained from the American Type Culture Collection (ATCC, Manassas, VA). The microsatellite status of each cell line was identified using labeled primers for the co-amplification of five quasimonomorphic mononucleotide repeat markers (BAT-25, BAT-26, NR-21, NR-24, and MONO-27) obtained from AmoyDx Biotechnology Co., Ltd (Xiamen, China). Consistent with existing literature reports (28), RKO and HCT-116 were classified as MSI-H, and HT-29 and SW-620 were classified as MSS. Cells were grown in Dulbecco's modified Eagle's medium/F12 medium supplemented with $10 \%$ fetal bovine serum and cultured in a humidified atmosphere containing $5 \% \mathrm{CO}_{2}$ at $37^{\circ} \mathrm{C}$.

\subsection{Western Blotting}

Cells were lysed with radioimmunoprecipitation assay buffer containing $1 \%$ phenylmethylsulfonyl fluoride. Insoluble materials were removed by centrifugation at $12,000 \mathrm{rpm}$ for $15 \mathrm{~min}$ at $4^{\circ} \mathrm{C}$. The concentration of total protein was determined using the Pierce BCA Protein Assay Kit (Thermo Fisher Scientific, Waltham, USA). Proteins were separated by $10 \%$ sodium dodecyl sulfate-polyacrylamide gel electrophoresis, transferred onto polyvinylidene difluoride membranes, and probed with the appropriate antibodies, as indicated. An antibody against mTOR (Rabbit, catalog number sc-1549-R) was obtained from Santa Cruz Biotechnology (Santa Cruz, CA, USA), and an antibody against p-mTOR (Rabbit, catalog number SAB4504476) was obtained from Sigma-Aldrich. An anti-glyceraldehyde 3-phosphate dehydrogenase (GAPDH; Mouse, catalog number 60004-1-lg) was purchased from Proteintech (Chicago, IL, USA). The secondary antibodies included anti-mouse (catalog number A4416) and anti-rabbit (catalog number A6154) antibodies obtained from Sigma-Aldrich.

\subsection{Cytotoxicity Experiments}


For cytotoxicity experiments, cell lines were seeded in a 96-well plate and treated with rapamycin (Med Chem Express, USA) for $72 \mathrm{~h}$. Rapamycin was dissolved in dimethylsulfoxide to a final concentration of $10 \mathrm{mM}$. The working concentrations were diluted to $0,10,15,20,25$, and $30 \mu \mathrm{M}$, and four wells were used for each concentration. Cell proliferation was assessed using the Cell Counting Kit-8 (CCK-8; Meilunbio Biotechnology Co., Ltd). The absorbance was measured at $450 \mathrm{~nm}$ using a model 3550 microplate reader (BioRad Laboratories, Inc., Hercules, CA, USA). Half maximal inhibitory concentration $\left(\mathrm{IC}_{50}\right)$ values were calculated, and the inhibition curve was plotted using GraphPad Prism software, Version 8.0.

\subsection{Patients and tissue samples}

Following the guidelines set by the Ethical Committee of Ruijin Hospital, 74 CRC cases were recruited from Ruijin Hospital (Shanghai, China). Clinicopathological data were retrospectively collected, comprising sex, age, tumor location, pathological tumor node metastasis stage, vascular invasion, and MLH1, PMS2, MSH2, and MSH6 expression (positive or negative). Informed consent was obtained from all patients before the study. The CRC stages were categorized according to Union for International Cancer Control guideline (8th Edition).

For the tissue microarray, the cohort of 74 tumor tissues and paired normal colonic tissues were fixed with formaldehyde and embedded with paraffin. A tissue microarray was constructed for further immunohistological assays. Based on the German semi-quantitative scoring system, the staining score for each tissue was evaluated by two independent pathologists, and a score greater than 3 was considered positive expression.

\subsection{DNA and Whole-exome sequence}

For whole-exome sequencing, DNA was isolated from 48 frozen tumor tissues collected from a previous cohort. DNA was isolated using standard extraction methods (Qiagen, Valencia, CA) and quantified using PicoGreen-based dsDNA detection (Life Technologies, Carlsbad, CA). Indexed sequencing libraries were prepared from $500 \mathrm{ng}$ sonically sheared DNA samples using Illumina TruSeq LT reagents (Illumina Inc, San Diego, CA).

Using a custom DNA bait set created by IDT (Integrated DNA Technologies, Coralville, lowa), libraries were enriched using solution-based hybrid capture. The DNA bait set included a target panel that covered the whole region of the MTOR gene, which encompasses 160,017 bp of the genome. Massively parallel sequencing was performed using an Illumina Novaseq6000 (Illumina) with paired-end 150 bp (PE150) reads.

Pooled sample reads were deconvoluted (demultiplexed) and sorted using Picard version 2.24.2 and later versions (Broad Institute, Cambridge, MA). Reads were aligned to the reference sequence hg19, obtained from the Human Genome Reference Consortium, using BWA version 0.7.17. Duplicate reads were identified and removed using Picard. The median mean target coverage per sample after the removal of duplicate reads was 1224 . The alignments were further refined using the Genome Analysis Toolkit version 4.1.1.0 and later versions (Broad Institute) for localized realignment around insertion and deletion 
(indel) sites. The recalibration of quality scores was performed using the Genome Analysis Toolkit. Mutation analysis for single-nucleotide variants was performed using MuTect (Broad Institute). Indels were called using Indelocator (Broad Institute). Integrative Genomics Viewer version 2.0.16 or later versions (Broad Institute) was used to visualize and interpret data. Variants were filtered to exclude synonymous variants, known germline variants in the Single-Nucleotide Polymorphism database, and variants that occur at a population frequency of $>0.1 \%$ in the Exome Sequencing Project database. Copy number detection was performed by analysis of fractional coverage of a defined genomic interval compared with pooled normal samples. The structural variant analysis was performed using Delly to detect larger indels. Finally, 10 cases were excluded from analysis due to disqualification of sequencing data, and 38 patients were selected for the final analysis.

\subsection{Statistical analysis}

R software was utilized for statistical analysis. The Pearson Chi-square test was used to analyze the association between MTOR mutations and clinical characteristic variables. A p-value $<0.05$ was considered significant.

\section{Results}

\subsection{MTOR expression analysis in the pan-cancer cohort indicated overexpression in CRC}

We first conducted a pan-cancer analysis of MTOR expression (Fig. 1a), and the TIMER analysis results indicated that compared with normal tissues, MTOR was downregulated in breast carcinoma (BRCA), clear cell RCC (KIRC), clear cell papillary RCC (KIRP), and chromophobe RCC (KICH), whereas MTOR was upregulated in cholangiocarcinoma (CHOL), esophageal carcinoma (ESCA), head and neck squamous cell carcinoma (HNSC), hepatocellular carcinoma (HCC), lung adenocarcinoma (LUAD), lung squamous cell carcinoma (LUSC), pancreatic adenocarcinoma (PAAD), gastric adenocarcinoma (STAD), and uterine corpus endometrial carcinoma (UCEC). The expression levels of MTOR in bladder urothelial carcinoma (BLCA), colon carcinoma (COAD), rectal carcinoma (READ), and thyroid carcinoma (THCA) did not differ from that in normal tissues. These results demonstrated that MTOR was abnormally expressed in multiple tumor types.

We determined the expression level of MTOR in CRC samples obtained from multiple TCGA datasets (TCGA-COAD combined with TCGA-READ). However, no difference in MTOR expression levels was observed between CRC and normal tissues (Fig. 1b). Ethnic differences can influence tumorigenesis; therefore, we selected CRC expression data obtained from an Asian population in the GEO database for further analysis. The expression of MTOR was significantly increased in unpaired CRC samples compared with normal tissues in the GSE41657 cohort ( $p=0.037$; Fig. 1c). The trend toward upregulation was also observed between paired CRC samples and normal samples in the GSE113513 cohort $(p<0.05$; Fig. 1d). Moreover, we collected $74 \mathrm{CRC}$ tumor tissues and paired normal mucosal tissue samples from 
patients with CRC for immunohistochemistry validation. The results showed that MTOR was significantly overexpressed in CRC tissues compared with normal mucosal tissues $(p<0.05$; Fig. 1e and $f)$.

Based on MTOR overexpression in Asian CRC populations, we further analyzed its prognostic value. First, we established low and high MTOR expression groups according to the median MTOR expression value. As indicated in Fig. 2a and c, no significant difference in prognosis between the two groups was observed in TCGA and GSE87211 datasets. We set the cutoff levels according to the quantile value (top $25 \%$ and bottom $25 \%$ ) to establish low and high MTOR expression groups. As shown in Fig. $2 \mathrm{~b}$, higher MTOR expression was significantly related to worse overall survival in the TCGA dataset $(p=0.012$, hazard ratio $=2.247,95 \%$ confidence interval $=1.194-4.226$ ). In the GEO database, although no significant difference was observed between the two groups, a trend toward worse prognosis was observed for the high MTOR expression group $(p=0.064$, hazard ratio $=2.845,95 \%$ confidence interval $=0.996-8.130)$.

\subsection{Predicted MTOR functions and pathways in CRC}

To clarify the underlying mechanism of MTOR in the promotion of CRC formation, we performed DEG analysis between high $(N=123)$ and low MTOR expression patients $(N=117$, determined by quartile) from the TCGA dataset. The heatmap and the volcano plot in Fig. 3a and $b$ display the up- and downregulated DEGs.

We also performed GO and KEGG pathway enrichment analyses on the DEGs. KEGG enrichment analysis showed that the DEGs were closely linked to a variety of pathways involved in viral transcription, cell adhesion, and translation (Fig. 3c). The GO analysis revealed several MF terms, including motor activity, cell adhesion molecule binding, cadherin binding, actin binding, and extracellular matrix structural constituents. GO BP terms indicated that DEGs were involved in viral transcription, nuclear-transcribed mRNA catabolic processes, cell-substrate junction assemblies, cell-substrate junction organizations, and cell junction assemblies. GO CC terms indicated various cellular structures, including cell-substrate junction, focal adhesion, ribosome, lamini-10 complex, and myosin II filament (Fig. 3d). Both GO and KEGG analyses identified MTOR as significantly correlated with cell adhesion and metabolism; therefore, we further explored the associations between MTOR and cell adhesion and translation through GSEA (Fig. 3e-I). Based on the validation dataset, we identified DEGs involved in metabolism pathways, including oxidative phosphorylation, fatty acid metabolism, and glutathione metabolism. These results showed that MTOR might affect these signaling pathways, contributing to a poor prognosis in CRC patients.

\subsection{Relationship between MTOR mRNA expression and somatic variants in CRC}

$\mathrm{CRC}$ is characterized by high levels of genetic mutation, which plays an important role in the response to therapy. To identify the somatic mutations among the 537 CRC patients in the TCGA database, mutation data were downloaded, and a summary of the mutation information was visualized in Fig. $4 \mathrm{a}$ and $\mathrm{b}$. The top 10 mutated genes in CRC by percentage are visualized, including APC (79\%), TP53 (61\%), TTN (47\%), 
MUC16 (25\%), SYNE1 (28\%), KRAS (42\%), FAT (22\%), RYR2 (19\%), OBSCN (18\%), and PI3KCA (25\%). Oncoplots displayed significantly mutated genes in the CRC cohort, which were sorted by mutational frequency. In addition, we explored the mutation information associated with mTOR pathway-related genes in CRC and found that the mutation frequency of MTOR was only inferior to the mutation frequencies of KRAS and PI3KCA (Fig. 4c).

Next, we assessed the distribution of somatic variants in CRC between the low and high MTOR expression groups (determined by quartile). No differences in classification were observed between the two groups, but the number of altered bases in each sample was significantly higher for the high MTOR expression group than for the low MTOR expression group (Fig. 5a and b). Analysis of the mutation annotation files for the TCGA cohort revealed that the mutation frequencies for the top 20 driver genes were significantly different between the low and high MTOR expression groups (Fig. $5 \mathrm{c}$ and d). In the high expression group, the mutation frequencies for the top 20 genes increased substantially, with all being greater than $20 \%$, whereas, in the low expression group, only eight genes had mutation frequencies greater than $20 \%$. We also positioned the TMB scores of the two groups within a pan-cancer TMB score scatter diagram, which revealed that the TMB of the high MTOR expression group was higher than the average TMB level of rectal cancer, whereas the TMB of the low MTOR expression group was lower than that of rectal cancer (Suppl. Fig. 1a and b). We also analyzed components of the affected pathways, including RAS, WNT, NOTCH, Hippo, MYC, TGF-beta, and other pathways associated with the development of colorectal cancer, and compared them between the low and high MTOR expression groups (Suppl. Fig. 1c and d). The mutational frequencies among the components of these pathways increased in the high MTOR expression group. We also employed the mafCompare function to identify differentially mutated genes between low and high MTOR expression groups. Comparison between the low and high MTOR expression groups revealed 27 genes that were differentially mutated $(p<0.001$; Fig. 5e). All of the identified genes were significantly enriched in the high MTOR expression group. The comparison of TMB between the two groups showed that the TMB level in the MTOR high expression group was higher than that of the low MTOR expression group $(\mathrm{p}<0.001 ; \mathrm{Fig}$. 5f). These results indicated that MTOR was closely related to tumor mutations.

\subsection{Relationship between MTOR and the tumor-infiltrating immune cells (TIICs) in CRC}

We employed TIMER to explore the relationships between MTOR expression and the infiltration levels of six immune cell subtypes in the CRC immune microenvironment. As shown in Fig. 6a, MTOR expression was significantly correlated with the infiltration levels of $B$ cells $(p=8.11 e-03 ; p=2.77 e-01), C D 4^{+} T$ cells $\left(p=2.35 e^{-19} ; p=7.88 e-03\right), C D 8^{+} T$ cells $(p=2.41 e-03 ; p=1.80 e-02)$, neutrophils $(p=2.73 e-12 ; p=$ $1.00 e-01)$, macrophages $(p=3.03 e-07 ; p=6.30 e-01)$, and dendritic cells $(p=1.50 e-10 ; p=1.10 e-03)$ in the COAD and READ databases, respectively. These results confirmed that MTOR expression was associated with the CRC immune microenvironment. To elucidate the potential mechanisms between MTOR expression and different immune cell infiltrations, we further analyzed the effects of somatic cell 
MTOR copy number alterations (CNAs) on immune cell infiltration (Fig. 6b). The MTOR CNAs included deep deletion, arm-level deletion, diploid/normal, and arm-level gain. Compared with diploid/normal, a decrease in the MTOR gene copy number (arm-level deletion) significantly alleviated the infiltration levels of $B$ cells $(p<0.001), C D 8^{+}$T cells $(p<0.001)$, neutrophils $(p<0.001)$, and dendritic cells $(p<0.001)$ in COAD. In READ, arm-level deletion in the MTOR gene copy number was associated with an increased infiltration level of $C D 4^{+} T$ cells $(p<0.05)$. These results demonstrated that MTOR had pivotal regulatory effects on the TME in CRC patients.

We used CIBERSORT to estimate the immune infiltration landscape of the TME by exploring the biological role of MTOR in the TME. The proportions of tumor-infiltrating immune cells (TIICs) in the TME varied significantly between low and high MTOR expression groups (Fig. 6c). The results further showed that $\mathrm{CD}^{+} \mathrm{T}$ cells, $\mathrm{T}$ regulatory (Treg) cells, follicular helper $\mathrm{T}$ cells, and resting dendritic cells were the primary immune cells affected by MTOR expression (Fig. 6d). Among these, CD $8^{+} T$ cells $(p<0.01)$, resting dendritic cells $(p<0.001)$, and Treg cells $(p<0.001)$ exhibited lower proportions in the high MTOR expression group than in the low MTOR expression group. Conversely, the proportion of follicular helper $T$ cells $(p<0.05)$ was significantly upregulated in the high MTOR expression group. In addition, we investigated the differences in the expression levels of immune checkpoints between the two groups (Fig. 6e). The results showed that expression of PDCD-1 (PD-1), CTLA4, LAG3, TNFRSF8/9/14/25, IDO2 et al were significantly different.

\subsection{Relationship between MTOR and MSI}

In addition to the comparison between CRC and normal tissue, we found that the expression of MTOR in the MSI subgroup was higher than that in the MSS subgroup (Fig. 7a). Analysis of mTOR protein levels using immunohistochemistry in CRC tissues revealed higher expression levels in the MSI samples than in the MSS samples (Fig. 7b). We performed western blot analysis to observe mTOR protein levels in different MSI and MSS CRC cell lines. The results showed that the protein levels of mTOR and p-mTOR were higher in MSI cell lines than in MSS cell lines (Fig. 7c). To investigate whether the activity of mTOR was changed in MSI CRC cell lines, we treated both MSI and MSS cell lines with rapamycin. As shown in Supplemental Fig. 2, cytotoxicity experiments revealed that rapamycin preferentially targeted MSI cell lines. MSS cell lines showed 2.4-fold and 1.4-fold higher $\mathrm{IC}_{50}$ values for rapamycin compared with the rapamycin $\mathrm{IC}_{50}$ values observed in MSI cell lines (22.68 vs. $9.57 \mu \mathrm{M}, 22.68$ vs. $16.01 \mu \mathrm{M}$, respectively). These results indicated that the activity of the mTOR signaling pathway might be altered in MSI CRC.

\subsection{Mutation signatures of MTOR in CRC}

Our results revealed that MTOR was a highly mutated gene in CRC (Fig. 4c). MTOR mutations have been shown to abnormally activate the mTOR pathway and increase sensitivity to rapamycin (29). To analyze MTOR mutations in CRC, we collected frozen tumor tissues from 48 patients with CRC and conducted whole-exome sequencing of MTOR genes. The clinicopathological data of this patient cohort are shown in Table 1.10 cases were excluded from analysis due to disqualification of sequencing data. A total of 38 
patients were able to be analyzed, including $15 \mathrm{MSI}-\mathrm{H}$ patients and $23 \mathrm{MSS}$ patients. MTOR mutations were identified by sequencing, classified, and analyzed. Among the classification categories, frameshift insertions and missense mutations accounted for the largest percentages of mutations, insertions occurred more frequently than single-nucleotide polymorphisms (SNP) or deletions, and T>A was the most common type of SNP in both the MSI-H and MSS groups (Fig. 8a and b). The frequencies of different mutational classes in MTOR were higher in the MSI-H group than in the MSS group (Fig. 8c-f). Among the variant classifications, the frequencies of frameshift deletions $(p<0.05)$, missense mutations $(p<0.05)$, nonsense mutations $(p<0.01)$, and splice sites $(p<0.01)$ were significantly increased in the MSI-H group compared with those in the MSS group. Among the variant types, the frequencies of SNPs ( $p$ $<0.05)$ and deletions $(p<0.05)$ were positive correlated with MSI. The analysis of SNPs showed that the proportion of $T>C$ transitions $(p<0.05)$ was significantly higher in the MSI-H group than in the MSS group. In the MSI-H group, the frequencies of all transitions were significantly higher than in the MSS group $(p<0.05)$, whereas the frequencies of transversions were lower than in the MSS group $(p<0.05)$. These results indicated that the mutation frequency of MTOR was higher among MSI-H patients than among MSS patients. 
Table 1

Baseline characteristic of patients with CRC from Ruijin Hospital $(\mathrm{N}=48)$

\begin{tabular}{|ll|}
\hline Factors & Cohort (N=48) \\
\hline Gender & \\
\hline Male & $29(60 \%)$ \\
\hline Female & $19(40 \%)$ \\
\hline Age & \\
\hline$<60$ y & $15(31 \%)$ \\
\hline$>60$ y & $33(69 \%)$ \\
\hline MS status & \\
\hline MSI-H & $17(35 \%)$ \\
\hline MSS & $31(65 \%)$ \\
\hline Tumor location & \\
\hline Right & $17(35 \%)$ \\
\hline Left & $31(65 \%)$ \\
\hline T stage & $30(63 \%)$ \\
\hline T2 & $3(6 \%)$ \\
\hline T3 & $23(48 \%)$ \\
\hline T4 & $22(46 \%)$ \\
\hline N stage & $36(75 \%)$ \\
\hline N0 & \\
\hline N1-2 & \\
\hline M stage & $(25 \%)$ \\
\hline M0 & \\
\hline M1 & \\
\hline Stage & \\
\hline I & \\
\hline II & \\
\hline
\end{tabular}

Page 13/29 


\begin{tabular}{|ll|}
\hline Factors & Cohort $(\mathrm{N}=\mathbf{4 8})$ \\
\hline IV & $7(14 \%)$ \\
\hline Vascular invasion & \\
\hline positive & $10(21 \%)$ \\
negative & $38(79 \%)$ \\
\hline
\end{tabular}

We also analyzed the relationship between MTOR mutations and clinicopathological signatures. In addition to a significant increase in the MSI-H group ( $\mathrm{p}=0.047$, Fig. 9a), the mutation frequency of MTOR differed according to tumor location. The mutation frequency of MTOR was higher in patients with rightsided CRC than in those with left-sided CRC ( $p=0.046$, Fig. 9c). A trend toward differences between MTOR mutation frequencies according to TN classification and tumor stage was observed, although no significant differences were identified, which may be due to the small sample size (Fig. 9e, f, and h). No significant differences in MTOR mutation frequencies were observed based on age, vascular invasion, or $M$ classification (Fig. 9b, d, and g).

\section{Discussion}

$\mathrm{CRC}$ is a highly heterogeneous disease caused by the interaction between genetic and environmental factors. Multiple alternative genetic pathways exist in CRC development. CRC evolves from benign to malignant lesions, and many mutations in oncogenes and tumor suppressor genes are involved in CRC tumorigenesis and progression $(30,31)$. CRC characterized by high mutation levels is thought to harbor an increased neoantigen burden, which is highly immunogenic and sensitive to immune checkpoint inhibitor (ICl) therapy (32). The investigation of predictive and prognostic biomarkers has historically focused on alterations in the RAS/BRAF/MEK/MAPK and PI3K/AKT/mTOR pathways. For the RAS/BRAF/MEK/MAPK pathway, fruitful and solid evidence has emerged supporting the use of BRAF as a prognostic biomarker and RAS as a predictive biomarker in CRC (33). However, biomarkers associated with the PI3K/AKT/mTOR pathway, such as PIK3CA and PTEN, are not recommended for use in clinical practice due to insufficient evidence, especially associated with CRC treatment $(34,35)$. Therefore, identifying other components of the PI3K/AKT/mTOR pathway that may serve as prognostic biomarkers for targeted therapy and ICI remains necessary. In this study, we focused on mTOR, which is a master regulator of the PI3K/AKT signaling pathway. The role of MTOR in CRC has been under-characterized, and we aimed to dissect its biological functions and reveal its associations with responses to targeted therapies.

The pan-cancer analysis showed that MTOR mRNA is overexpressed in many cancers. However, analysis of RNA sequencing data from TCGA (COAD and READ) suggested no significant differences in mTOR expression between tumor tissues and normal tissues, which was inconsistent with previous findings (20-22). Considering the potential for ethnic differences to affect tumorigenesis, we further selected expression data associated with CRC among an Asian population from the GEO database for analysis 
and identified that the expression of MTOR in tumor tissues was upregulated compared with normal tissues in an ethnicity-dependent manner in an Asian cohort.

To analyze the prognostic value of MTOR among CRC patients, we divided CRC patients from the TCGA dataset into high and low MTOR expression groups based on the median and quartile levels. No significant difference was expected between groups based on the median level because the expression of $M T O R$ in cancer tissues did not differ significantly from that in normal tissues. However, when analyzed according to the quartile level (top 25\% and bottom 25\%), the high MTOR expression group was significantly associated with poor survival, which suggested that differences in MTOR expression level were associated with prognosis. A similar trend was also found among CRC patients from the GEO database, although the differences in the GEO dataset did not reach significance, possibly due to the small sample size. These results indicated the potential for MTOR to serve as a prognostic marker in Asian CRC patients, although this possibility requires further clinical validation.

Pathway analysis and GSEA results showed that DEGs were enriched in the metabolism, cell adhesion, and translation pathways. MTOR was associated with key fatty acid, glutathione, and oxidative phosphorylation metabolic pathways in CRC. Fatty acids are indispensable for the synthesis of membranes and signaling molecules, which are associated with cell proliferation (36). As an antioxidant, glutathione has profound effects on cell survival while also conferring therapeutic resistance to cancer cells (37). Oxidative phosphorylation is a primary source of energy. Studies have shown that mitochondrial DNA (mtDNA) content is higher in CRC than in normal tissue, which may indicate a higher contribution of oxidative phosphorylation in CRC (38). MTOR serves as a key regulator of these metabolic pathways (39-41). In addition, MTOR regulates protein translation and synthesis to promote cell proliferation. The proliferation of CRC cells can be disrupted by reducing the phosphorylation level of elF4E-binding protein 1 (4EBP1), which is a tumor suppressor protein activated by mTOR (42). Cell adhesion is a key mediator of cancer progression and facilitates several behavioral hallmarks of cancer, including immune evasion and metastatic dissemination (43). Further study found that mTOR complex (mTORC) 1 and mTORC2 were both involved in the regulation of cell adhesion (44). Therefore, MTOR might affect these signaling pathways, contributing to a poor prognosis in CRC patients

Our study revealed that MTOR expression was associated with a high somatic mutation burden. In theory, tumors with a higher number of genetic variations are statistically more likely to generate novel mutant proteins or neoantigens, which may be recognized as foreign invaders by the immune system and trigger a cytotoxic, tumor-killing response (45). Relevant analyses revealed a correlation between TMB and the response rates and outcomes of $\mathrm{ICl}$ therapy (46). ICl therapy has shown promising results in various types of cancers, particularly antibodies against the programmed cell death protein 1 (PD-1) T-cell coreceptor and its ligand B7-H1/programmed death-ligand 1 (PD-L1), which have induced durable tumor responses, even in late-stage patients who have failed to respond to multiple classical treatment strategies $(47,48)$. Previous studies have indicated that immune infiltration levels are related to prognosis and the response to $\mathrm{ICl}$ therapy for several cancers, including esophageal squamous cell carcinoma, breast cancer, and CRC (49-51). In the TME, we found that the MTOR expression and CNAs significantly 
affected the immune cell infiltration of $\mathrm{CD} 8^{+} \mathrm{T}$ cells, B cells, neutrophils, and dendritic cells in CRC. Using CIBERSORT, our results showed that $\mathrm{CD} 8^{+} \mathrm{T}$ cells were negatively correlated with MTOR expression. In addition, we found that the expression levels of PDCD-1, CTLA4, LAG3 were positively associated with MTOR expression. These results indicated that MTOR status could potentially be used to assess whether patients might benefit from ICl therapy.

Moreover, our results showed that the expression and function of MTOR were altered in MSI-H CRC patients. MSI is an intensively studied biomarker with prognostic and therapeutic values in CRC. MSI refers to changes in the lengths of short-tandem-repeat DNA sequences, the presence of which represents phenotypic evidence of deficient mismatch repair (dMMR). MMR is a highly conserved cellular process intended to correct erroneous insertions, deletions, and base-base mismatches that occur during DNA replication and recombination and have escaped the proofreading process. When the MMR system develops a malfunction, errors generated during DNA replication increase, including single-base substitutions, insertions, or deletions of short-tandem-repeat DNA sequences, resulting in MSI (52). MSI is present in approximately $15 \%$ of CRC and $5 \%$ of metastatic CRC (mCRC) $(53,54)$. Depending on the degree of instability, MSI tumors can be divided into MSI-H or MSI-L subsets. Several clinical studies have found that patients with MSI-H present with a durable and robust response to ICI therapy $(55,56)$. Although the response rates to $\mathrm{ICl}$ among patients with MSI-H CRC have been variable in different trials, more somatic mutations and higher neoantigen burdens were identified in responsive tumors than nonresponsive tumors (57).

Few studies have reported the correlation between MTOR and MSI-H. Vilar et al. (58) reported that MSI-H cell lines responded better to therapies that preferentially target the PI3K/AKT/mTOR pathway but did not explore the expression of MTOR in MSI-H. Consistent with these results, we revealed that rapamycin preferentially targeted MSI-H cell lines via cytotoxicity experiments. Additionally, we found that the expression of MTOR increased in MSI-H CRC samples from a public database, which was then validated in both cell lines and CRC cohorts from our center. Lin et al. reported that MSI-H CRC tumors feature a significantly increased number of mTOR pathway mutations than MSS tumors (59). In addition, evidence has shown that MTOR mutants can constitutively activate the mTOR signaling pathway and increase the sensitivity to rapalog treatment (60). Therefore, whole-exome sequencing in CRC patients (MSI-H or MSS) was applied to detect variations among all SNP sites in MTOR. To the best of our knowledge, this is the first systematic study examining the relationship between MTOR mutations and CRC. Our analysis showed that the mutation frequency of MTOR in MSI-H patients was significantly increased compared with MSS patients, which can affect the protein activity of mTOR and influence the tumor response to rapamycin treatment. Furthermore, we observed a significant increase in the frequency of transition mutations in the MSI-H group compared with that in the MSS group, which may be attributed to dMMR. Studies on MMR function have shown that MMR has a higher repair efficiency for transition mutations than other mutations $(61,62)$. These results suggested that MTOR mutations may present at a higher frequency in CRC patients with dMMR. Combined with the relationship between MTOR and high TMB, 
MTOR status could potentially be used to assess whether MSI-H patients might benefit from ICl therapy. Further research examining the efficacy of ICI therapy in MTOR-mutant MSI-H CRC remains necessary.

In this study, we found that MTOR played an important role in tumorigenesis and was associated with the immunological status of CRC. The function of MTOR in immunity has been extensively studied. Several studies have reported that the mTOR signaling pathway affects the function of immune cells and cytokines in the TME (63-65). In our study, MTOR was shown to be associated with the infiltration of various immune cells. Moreover, our study found that the high expression of MTOR was related to high TMB. Tumors with higher numbers of genetic variations are more likely to generate novel mutant proteins or neoantigens. Therefore, MTOR is likely not only associated with immune cell function but also with tumor cell immunogenicity, suggesting that MTOR may play a central role in tumor immunity. Interestingly, we found that MTOR was associated with MSI status, which was characterized by high TMB and abundant TIICs. The consistent relationship identified between MTOR mutations and MSI suggests that MTOR may represent a marker for the prediction of MSI status and tumor immunogenicity. Yang et al. (66) reported that MSI CRC tumors had higher expression levels of thymocyte selection-associated high-mobility group box (TOX), an inhibitor of mTOR, compared with MSS tumors. Our research focused on MTOR mutations rather than MTOR expression levels, and the work by Yang et al. did not examine the relationship between TOX expression and MTOR mutation.

The significant contributions of MTOR to CRC tumorigenesis suggest that mTOR inhibitors may be effective for CRC therapy. Previous clinical trials have studied the efficacy of temsirolimus and everolimus in mCRC patients. For example, in a clinical phase I/II study, everolimus combined with mFOLFOX-6 and bevacizumab was found to be tolerable and demonstrated preliminary efficacy for mCRC therapy. The objective response rate was $53 \%$ in $\mathrm{mCRC}$ patients and was higher ( $86 \%$ ) in those cases with PTEN deficiency (67). Studies on other types of inhibitors, such as ATP-competitive mTOR inhibitors and mTOR/PI3K dual inhibitors, have also shown tumor growth inhibition effects against CRC cell lines and xenograft models $(68,69)$. Moreover, studies have found that BEZ235, an mTOR/PI3K dual inhibitor, was capable of inducing a treatment response and overcoming resistance to everolimus in $A P C$ and PIK3CA-mutant CRC cells $(70,71)$. These findings suggested that different molecular subtypes might be associated with mTOR inhibition responses, which could be used to distinguish patients who will benefit from mTOR inhibition therapy. Our results revealed MTOR as an oncogene, and its mutation frequency in MSI-H patients was significantly higher than that in the MSS group. Further research examining the efficacy of mTOR inhibitors in MTOR-mutant CRC remains necessary. Moreover, the status of MTOR has been shown to regulate immunoreactions. Rapamycin is widely used as an immunosuppressant to prevent immune rejection in kidney transplant patients. Jung et al. found that rapamycin uniquely enhanced the number and function of $\mathrm{CD} 8^{+}$effector and central memory $\mathrm{T}$ cells (Jung et al., 2018). In a mouse model of RCC, anti-PD-L1 combined with everolimus was more effective for tumor regression than individual treatment due to the upregulation of PD-L1 in tumor cells, which increased the tumor-infiltrating $\mathrm{CD} 8^{+} \mathrm{T}$ cells (73). Similarly, our study demonstrated that the status of MTOR could be used to assess the immunological function of CRC patients and might serve as a 
potential indicator that can predict the optimum response to ICl therapy. mTOR inhibitors that promote cancer cell death and boost effector functions in T cells can be combined to improve ICl therapeutic outcomes. The relationship between MTOR mutations and dMMR suggests that CRC patients with dMMR are likely to benefit from combination therapy.

\section{Conclusion}

In conclusion, our study found that MTOR plays an important role in CRC tumorigenesis and was associated with prognosis, TMB, TIICs, and MSI status. Therefore, MTOR may represent a comprehensive indicator of prognosis and immunological status for CRC. Moreover, the mutation profile of MTOR may provide guidance for subsequent clinical research on mTOR inhibitors in CRC.

\section{Abbreviations}




\begin{tabular}{|c|c|}
\hline PI3K & phosphoinositide 3-kinase \\
\hline AKT & protein kinase B \\
\hline RCC & renal cell carcinoma \\
\hline $\mathrm{CRC}$ & colorectal cancer \\
\hline PTEN & phosphatase and tensin homolog \\
\hline PIK3CA & phosphatidylinositol-4,5-bisphosphate 3-kinase catalytic subunit alpha \\
\hline TMB & tumor mutational burden \\
\hline MSI & microsatellite instability \\
\hline MSI-H & MSI-high \\
\hline MSI-L & MSI-low \\
\hline MSS & microsatellite stable \\
\hline TCGA & The Cancer Genome Atlas \\
\hline GEO & Gene Expression Omnibus \\
\hline GO & Gene Ontology \\
\hline KEGG & Kyoto Encyclopedia of Genes and Genomes \\
\hline GSEA & gene set enrichment analysis \\
\hline COAD & colon carcinoma \\
\hline READ & rectal carcinoma \\
\hline TIMER & Tumor Immune Estimation Resource \\
\hline DEGs & differentially expressed genes \\
\hline TME & tumor microenvironment \\
\hline $\mathrm{IC}_{50}$ & half maximal inhibitory concentration \\
\hline TIICs & tumor-infiltrating immune cells \\
\hline CNAs & copy number alterations \\
\hline SNP & single-nucleotide polymorphisms \\
\hline $\mathrm{ICl}$ & immune checkpoint inhibitor \\
\hline
\end{tabular}

\section{Declarations}




\section{Ethics approval and consent to participate}

The studies involving human participants were reviewed and approved by the Ethical Committee of Ruijin Hospital, Shanghai Jiao Tong University School of Medicine. The patients/participants provided their written informed consent to participate in this study.

\section{Consent for publication}

Not applicable

\section{Availability of data and materials}

The raw sequence data reported in this paper have been deposited in the Genome Sequence Archive (74) in National Genomics Data Center (75), China National Center for Bioinformation / Beijing Institute of Genomics, Chinese Academy of Sciences, under accession number HRA000775 that are publicly accessible at https://bigd.big.ac.cn/gsa.

\section{Competing interests}

The authors declare that they have no competing interests.

\section{Funding}

This work was supported by Shanghai Municipal Education Commission, Gaofeng Clinical Medicine Grant Support, Shanghai Jiao Tong University School of Medicine (Grant No. 826304); National Nature Science Foundation of China (NSFC) (Grant No. 81871984); and Wu Jieping Medical Foundation (Grant No. 320.6750.14321).

\section{Author Contributions}

Conceptualization, Y.S. and J.S.; Data curation, B.A.; Formal analysis, Y.S.; Funding acquisition, J.L. and M.Z.; Investigation, C.W.; Methodology, C.W. and Y.S.; Project administration, S.Z. and J.S.; Resources, B.A. and H.J.; Software, Y.S. and X.Y.; Super-vision, J.L.; Validation, C.W.; Visualization, L.H. and H.H.; Writing original draft, C.W. and Y.S.; Writing - review \& editing, B.A., G.Y. and J.S..All authors read and approved the final manuscript.

\section{Acknowledgments}

We would like to acknowledge our lab colleagues for their help in analysis and experiments.

\section{References}

1. Weichhart T. mTOR as Regulator of Lifespan, Aging, and Cellular Senescence: A Mini-Review. Gerontology. 2018;64(2):127-34. doi: 10.1159/000484629. 
2. Murugan AK. mTOR: Role in cancer, metastasis and drug resistance. Semin Cancer Biol. 2019;59:92111. doi: 10.1016/j.semcancer.2019.07.003.

3. Forbes SA, Bindal N, Bamford S, Cole C, Kok CY, Beare D, et al. COSMIC: mining complete cancer genomes in the Catalogue of Somatic Mutations in Cancer. Nucleic Acids Res. 2011;39(Database issue):D945-50. doi: 10.1093/nar/gkq929.

4. Dhillon T, Mauri FA, Bellezza G, Cagini L, Barbareschi M, North BV, et al. Overexpression of the mammalian target of rapamycin: a novel biomarker for poor survival in resected early stage nonsmall cell lung cancer. J Thorac Oncol. 2010;5(3):314-9. doi: 10.1097/JT0.0b013e3181ce6604.

5. Gately K, Al-Alao B, Dhillon T, Mauri F, Cuffe S, Seckl M, et al. Overexpression of the mammalian target of rapamycin (mTOR) and angioinvasion are poor prognostic factors in early stage NSCLC: a verification study. Lung Cancer. 2012;75(2):217-22. doi: 10.1016/j.lungcan.2011.06.012.

6. Wazir U, Newbold RF, Jiang WG, Sharma AK, Mokbel K. Prognostic and therapeutic implications of mTORC1 and Rictor expression in human breast cancer. Oncol Rep. 2013;29(5):1969-74. doi: 10.3892/or.2013.2346.

7. Walsh S, Flanagan L, Quinn C, Evoy D, McDermott EW, Pierce A, et al. mTOR in breast cancer: differential expression in triple-negative and non-triple-negative tumors. Breast. 2012;21(2):178-82. doi: 10.1016/j.breast.2011.09.008.

8. Graham L, Banda K, Torres A, Carver BS, Chen Y, Pisano K, et al. A phase Il study of the dual mTOR inhibitor MLN0128 in patients with metastatic castration resistant prostate cancer. Invest New Drugs. 2018;36(3):458-67. doi: 10.1007/s10637-018-0578-9.

9. Mateo J, Olmos D, Dumez H, Poondru S, Samberg NL, Barr S, et al. A first in man, dose-finding study of the mTORC1/mTORC2 inhibitor OSI-027 in patients with advanced solid malignancies. $\mathrm{Br} \mathrm{J}$ Cancer. 2016;114(8):889-96. doi: 10.1038/bjc.2016.59.

10. Bendell JC, Kurkjian C, Infante JR, Bauer TM, Burris HA, 3rd, Greco FA, et al. A phase 1 study of the sachet formulation of the oral dual PI3K/mTOR inhibitor BEZ235 given twice daily (BID) in patients with advanced solid tumors. Invest New Drugs. 2015;33(2):463-71. doi: 10.1007/s10637-015-0218-6.

11. Rodon J, Pérez-Fidalgo A, Krop IE, Burris H, Guerrero-Zotano A, Britten CD, et al. Phase 1/1b dose escalation and expansion study of BEZ235, a dual PI3K/mTOR inhibitor, in patients with advanced solid tumors including patients with advanced breast cancer. Cancer Chemother Pharmacol. 2018;82(2):285-98. doi: 10.1007/s00280-018-3610-z.

12. Massard C, Chi KN, Castellano D, de Bono J, Gravis G, Dirix L, et al. Phase lb dose-finding study of abiraterone acetate plus buparlisib (BKM120) or dactolisib (BEZ235) in patients with castrationresistant prostate cancer. Eur J Cancer. 2017;76:36-44. doi: 10.1016/j.ejca.2017.01.024.

13. Hudes G, Carducci M, Tomczak P, Dutcher J, Figlin R, Kapoor A, et al. Temsirolimus, interferon alfa, or both for advanced renal-cell carcinoma. N Engl J Med. 2007;356(22):2271-81. doi: 10.1056/NEJMoa066838.

14. Iyer G, Hanrahan AJ, Milowsky MI, Al-Ahmadie H, Scott SN, Janakiraman M, et al. Genome sequencing identifies a basis for everolimus sensitivity. Science. 2012;338(6104):221. doi: 
10.1126/science.1226344.

15. Voss MH, Hakimi AA, Pham CG, Brannon AR, Chen YB, Cunha LF, et al. Tumor genetic analyses of patients with metastatic renal cell carcinoma and extended benefit from mTOR inhibitor therapy. Clin Cancer Res. 2014;20(7):1955-64. doi: 10.1158/1078-0432.ccr-13-2345.

16. Wagle N, Grabiner BC, Van Allen EM, Amin-Mansour A, Taylor-Weiner A, Rosenberg M, et al. Response and acquired resistance to everolimus in anaplastic thyroid cancer. $\mathrm{N}$ Engl $\mathrm{J}$ Med. 2014;371(15):1426-33. doi: 10.1056/NEJMoa1403352.

17. Bray F, Ferlay J, Soerjomataram I, Siegel RL, Torre LA, Jemal A. Global cancer statistics 2018 : GLOBOCAN estimates of incidence and mortality worldwide for 36 cancers in 185 countries. CA Cancer J Clin. 2018;68(6):394-424. doi: 10.3322/caac.21492.

18. Zheng RS, Sun KX, Zhang SW, Zeng HM, Zou XN, Chen R, et al. [Report of cancer epidemiology in China, 2015]. Zhonghua Zhong Liu Za Zhi. 2019;41(1):19-28. doi: 10.3760/cma.j.issn.02533766.2019.01.005.

19. Miller KD, Nogueira L, Mariotto AB, Rowland JH, Yabroff KR, Alfano CM, et al. Cancer treatment and survivorship statistics, 2019. CA Cancer J Clin. 2019;69(5):363-85. doi: 10.3322/caac.21565.

20. Fu-rong Y, Ke-yu P, Li Z. The expression of PI3K/AKT/mTOR pathway in colorectal cancer and its targeted therapy. Modern Digestion and Intervention. 2020;25(2):5. doi: 10.3969/j.issn.16722159.2020.02.010.

21. Yue Feng Y-tZ, Li-feng Xia, Yong-gang Ma. Expression of protein associated with PI3K/Akt/mTOR pathway and its relationship with clinical pathology and prognosis in colorectal cancer. China Journal of Modern Medicine. 2020;30(24):6. doi: 10.3969/j.issn.1005-8982.2020.24.004.

22. Xing W, Wei-dong S, Yong-ping M, Yong-gang Z. Expression of mTOR and PTEN in colorectal carcinoma and its clinical significance. Occupy and Health. 2015;31(8):4. doi:

10.13329/j.cnki.zyyjk.2015.0314.

23. Gulhati P, Cai Q, Li J, Liu J, Rychahou PG, Qiu S, et al. Targeted inhibition of mammalian target of rapamycin signaling inhibits tumorigenesis of colorectal cancer. Clin Cancer Res. 2009;15(23):720716. doi: 10.1158/1078-0432.ccr-09-1249.

24. Li T, Fan J, Wang B, Traugh N, Chen Q, Liu JS, et al. TIMER: A Web Server for Comprehensive Analysis of Tumor-Infiltrating Immune Cells. Cancer Res. 2017;77(21):e108-e10. doi: 10.1158/0008-5472.can17-0307.

25. Blum A, Wang P, Zenklusen JC. SnapShot: TCGA-Analyzed Tumors. Cell. 2018;173(2):530. doi: 10.1016/j.cell.2018.03.059.

26. Mayakonda A, Lin DC, Assenov Y, Plass C, Koeffler HP. Maftools: efficient and comprehensive analysis of somatic variants in cancer. Genome Res. 2018;28(11):1747-56. doi:

10.1101/gr.239244.118.

27. Newman AM, Liu CL, Green MR, Gentles AJ, Feng W, Xu Y, et al. Robust enumeration of cell subsets from tissue expression profiles. Nat Methods. 2015;12(5):453-7. doi: 10.1038/nmeth.3337. 
28. Ahmed D, Eide PW, Eilertsen IA, Danielsen SA, Eknæs M, Hektoen M, et al. Epigenetic and genetic features of 24 colon cancer cell lines. Oncogenesis. 2013;2(9):e71. doi: 10.1038/oncsis.2013.35.

29. Yamaguchi H, Kawazu M, Yasuda T, Soda M, Ueno T, Kojima S, et al. Transforming somatic mutations of mammalian target of rapamycin kinase in human cancer. Cancer Sci. 2015;106(12):1687-92. doi: 10.1111/cas.12828.

30. Fearon ER, Vogelstein B. A genetic model for colorectal tumorigenesis. Cell. 1990;61(5):759-67. doi: 10.1016/0092-8674(90)90186-i.

31. Neumann J, Wehweck L, Maatz S, Engel J, Kirchner T, Jung A. Alterations in the EGFR pathway coincide in colorectal cancer and impact on prognosis. Virchows Arch. 2013;463(4):509-23. doi: 10.1007/s00428-013-1450-0.

32. McGranahan N, Furness AJ, Rosenthal R, Ramskov S, Lyngaa R, Saini SK, et al. Clonal neoantigens elicit T cell immunoreactivity and sensitivity to immune checkpoint blockade. Science. 2016;351(6280):1463-9. doi: 10.1126/science.aaf1490.

33. Taieb J, Jung A, Sartore-Bianchi A, Peeters M, Seligmann J, Zaanan A, et al. The Evolving Biomarker Landscape for Treatment Selection in Metastatic Colorectal Cancer. Drugs. 2019;79(13):1375-94. doi: 10.1007/s40265-019-01165-2.

34. Van Cutsem E, Cervantes A, Adam R, Sobrero A, Van Krieken JH, Aderka D, et al. ESMO consensus guidelines for the management of patients with metastatic colorectal cancer. Ann Oncol. 2016;27(8):1386-422. doi: 10.1093/annonc/mdw235.

35. Sepulveda AR, Hamilton SR, Allegra CJ, Grody W, Cushman-Vokoun AM, Funkhouser WK, et al. Molecular Biomarkers for the Evaluation of Colorectal Cancer: Guideline From the American Society for Clinical Pathology, College of American Pathologists, Association for Molecular Pathology, and American Society of Clinical Oncology. J Mol Diagn. 2017;19(2):187-225. doi: 10.1016/j.jmoldx.2016.11.001.

36. Currie E, Schulze A, Zechner R, Walther TC, Farese RV, Jr. Cellular fatty acid metabolism and cancer. Cell Metab. 2013;18(2):153-61. doi: 10.1016/j.cmet.2013.05.017.

37. Bansal A, Simon MC. Glutathione metabolism in cancer progression and treatment resistance. J Cell Biol. 2018;217(7):2291-8. doi: 10.1083/jcb.201804161.

38. Yu M. Generation, function and diagnostic value of mitochondrial DNA copy number alterations in human cancers. Life Sci. 2011;89(3-4):65-71. doi: 10.1016/j.Ifs.2011.05.010.

39. Mossmann D, Park S, Hall MN. mTOR signalling and cellular metabolism are mutual determinants in cancer. Nat Rev Cancer. 2018;18(12):744-57. doi: 10.1038/s41568-018-0074-8.

40. Morita M, Gravel SP, Hulea L, Larsson O, Pollak M, St-Pierre J, et al. mTOR coordinates protein synthesis, mitochondrial activity and proliferation. Cell Cycle. 2015;14(4):473-80. doi: 10.4161/15384101.2014.991572.

41. Kim SG, Lee SJ. PI3K, RSK, and mTOR signal networks for the GST gene regulation. Toxicol Sci. 2007;96(2):206-13. doi: 10.1093/toxsci/kfl175. 
42. Alqurashi N, Hashimi SM, Alowaidi F, Ivanovski S, Wei MQ. Dual mTOR/PI3K inhibitor NVP-BEZ235 arrests colorectal cancer cell growth and displays differential inhibition of 4E-BP1. Oncol Rep. 2018;40(2):1083-92. doi: 10.3892/or.2018.6457.

43. Läubli H, Borsig L. Altered Cell Adhesion and Glycosylation Promote Cancer Immune Suppression and Metastasis. Front Immunol. 2019;10:2120. doi: 10.3389/fimmu.2019.02120.

44. Chen L, Xu B, Liu L, Liu C, Luo Y, Chen X, et al. Both mTORC1 and mTORC2 are involved in the regulation of cell adhesion. Oncotarget. 2015;6(9):7136-50. doi: 10.18632/oncotarget.3044.

45. Samstein RM, Lee CH, Shoushtari AN, Hellmann MD, Shen R, Janjigian YY, et al. Tumor mutational load predicts survival after immunotherapy across multiple cancer types. Nat Genet. 2019;51(2):2026. doi: 10.1038/s41588-018-0312-8.

46. Yarchoan M, Hopkins A, Jaffee EM. Tumor Mutational Burden and Response Rate to PD-1 Inhibition. N Engl J Med. 2017;377(25):2500-1. doi: 10.1056/NEJMc1713444.

47. Brahmer JR, Tykodi SS, Chow LQ, Hwu WJ, Topalian SL, Hwu P, et al. Safety and activity of anti-PDL1 antibody in patients with advanced cancer. N Engl J Med. 2012;366(26):2455-65. doi: 10.1056/NEJMoa1200694.

48. Topalian SL, Hodi FS, Brahmer JR, Gettinger SN, Smith DC, McDermott DF, et al. Safety, activity, and immune correlates of anti-PD-1 antibody in cancer. N Engl J Med. 2012;366(26):2443-54. doi: 10.1056/NEJMoa1200690.

49. Baba $Y$, Nomoto D, Okadome $K$, Ishimoto T, Iwatsuki M, Miyamoto $Y$, et al. Tumor immune microenvironment and immune checkpoint inhibitors in esophageal squamous cell carcinoma. Cancer Sci. 2020;111(9):3132-41. doi: 10.1111/cas.14541.

50. Zhou Z, Xie X, Wang X, Zhang X, Li W, Sun T, et al. Correlations Between Tumor Mutation Burden and Immunocyte Infiltration and Their Prognostic Value in Colon Cancer. Front Genet. 2021;12:623424. doi: $10.3389 /$ fgene.2021.623424.

51. Karn T, Denkert C, Weber KE, Holtrich U, Hanusch C, Sinn BV, et al. Tumor mutational burden and immune infiltration as independent predictors of response to neoadjuvant immune checkpoint inhibition in early TNBC in GeparNuevo. Ann Oncol. 2020;31(9):1216-22. doi:

10.1016/j.annonc.2020.05.015.

52. Baretti M, Le DT. DNA mismatch repair in cancer. Pharmacol Ther. 2018;189:45-62. doi: 10.1016/j.pharmthera.2018.04.004.

53. Ionov Y, Peinado MA, Malkhosyan S, Shibata D, Perucho M. Ubiquitous somatic mutations in simple repeated sequences reveal a new mechanism for colonic carcinogenesis. Nature. 1993;363(6429):558-61. doi: 10.1038/363558a0.

54. De' Angelis GL, Bottarelli L, Azzoni C, De' Angelis N, Leandro G, Di Mario F, et al. Microsatellite instability in colorectal cancer. Acta Biomed. 2018;89(9-s):97-101. doi: 10.23750/abm.v89i9-S.7960.

55. Overman MJ, McDermott R, Leach JL, Lonardi S, Lenz HJ, Morse MA, et al. Nivolumab in patients with metastatic DNA mismatch repair-deficient or microsatellite instability-high colorectal cancer 
(CheckMate 142): an open-label, multicentre, phase 2 study. Lancet Oncol. 2017;18(9):1182-91. doi: 10.1016/s1470-2045(17)30422-9.

56. Le DT, Kim TW, Van Cutsem E, Geva R, Jäger D, Hara H, et al. Phase II Open-Label Study of Pembrolizumab in Treatment-Refractory, Microsatellite Instability-High/Mismatch Repair-Deficient Metastatic Colorectal Cancer: KEYNOTE-164. J Clin Oncol. 2020;38(1):11-9. doi: $10.1200 /$ jco.19.02107.

57. Le DT, Durham JN, Smith KN, Wang H, Bartlett BR, Aulakh LK, et al. Mismatch repair deficiency predicts response of solid tumors to PD-1 blockade. Science. 2017;357(6349):409-13. doi: 10.1126/science.aan6733.

58. Vilar E, Mukherjee B, Kuick R, Raskin L, Misek DE, Taylor JM, et al. Gene expression patterns in mismatch repair-deficient colorectal cancers highlight the potential therapeutic role of inhibitors of the phosphatidylinositol 3-kinase-AKT-mammalian target of rapamycin pathway. Clin Cancer Res. 2009;15(8):2829-39. doi: 10.1158/1078-0432.ccr-08-2432.

59. Lin El, Tseng LH, Gocke CD, Reil S, Le DT, Azad NS, et al. Mutational profiling of colorectal cancers with microsatellite instability. Oncotarget. 2015;6(39):42334-44. doi: 10.18632/oncotarget.5997.

60. Grabiner BC, Nardi V, Birsoy K, Possemato R, Shen K, Sinha S, et al. A Diverse Array of CancerAssociated MTOR Mutations Are Hyperactivating and Can Predict Rapamycin Sensitivity. Cancer Discovery. 2014;4(5):554-63. doi: 10.1158/2159-8290.Cd-13-0929.

61. Jones $M$, Wagner R, Radman M. Repair of a mismatch is influenced by the base composition of the surrounding nucleotide sequence. Genetics. 1987;115(4):605-10.

62. Lujan SA, Williams JS, Pursell ZF, Abdulovic-Cui AA, Clark AB, Nick McElhinny SA, et al. Mismatch repair balances leading and lagging strand DNA replication fidelity. PLoS Genet. 2012;8(10):e1003016. doi: 10.1371/journal.pgen.1003016.

63. Rostamzadeh D, Yousefi M, Haghshenas MR, Ahmadi M, Dolati S, Babaloo Z. mTOR Signaling pathway as a master regulator of memory CD8(+) T-cells, Th17, and NK cells development and their functional properties. J Cell Physiol. 2019;234(8):12353-68. doi: 10.1002/jcp.28042.

64. Bazzichetto C, Conciatori F, Falcone I, Ciuffreda L. Translational Landscape of mTOR Signaling in Integrating Cues Between Cancer and Tumor Microenvironment. Adv Exp Med Biol. 2020;1223:69-80. doi: 10.1007/978-3-030-35582-1_4.

65. Zeng H. mTOR signaling in immune cells and its implications for cancer immunotherapy. Cancer Lett. 2017;408:182-9. doi: 10.1016/j.canlet.2017.08.038.

66. Yang M, Huang Q, Li C, Jiang Z, Sun J, Wang Z, et al. TOX Acts as a Tumor Suppressor by Inhibiting mTOR Signaling in Colorectal Cancer. Front Immunol. 2021;12:647540. doi: 10.3389/fimmu.2021.647540.

67. Weldon Gilcrease G, Stenehjem DD, Wade ML, Weis J, McGregor K, Whisenant J, et al. Phase I/II study of everolimus combined with mFOLFOX- 6 and bevacizumab for first-line treatment of metastatic colorectal cancer. Invest New Drugs. 2019;37(3):482-9. doi: 10.1007/s10637-018-0645-2. 
68. Bhagwat SV, Gokhale PC, Crew AP, Cooke A, Yao Y, Mantis C, et al. Preclinical characterization of OSI027, a potent and selective inhibitor of mTORC1 and mTORC2: distinct from rapamycin. Mol Cancer Ther. 2011;10(8):1394-406. doi: 10.1158/1535-7163.mct-10-1099.

69. Roper J, Richardson MP, Wang WV, Richard LG, Chen W, Coffee EM, et al. The dual PI3K/mTOR inhibitor NVP-BEZ235 induces tumor regression in a genetically engineered mouse model of PIK3CA wild-type colorectal cancer. PLoS One. 2011;6(9):e25132. doi: 10.1371/journal.pone.0025132.

70. Fricke SL, Payne SN, Favreau PF, Kratz JD, Pasch CA, Foley TM, et al. MTORC1/2 Inhibition as a Therapeutic Strategy for PIK3CA Mutant Cancers. Mol Cancer Ther. 2019;18(2):346-55. doi: 10.1158/1535-7163.mct-18-0510.

71. Foley TM, Payne SN, Pasch CA, Yueh AE, Van De Hey DR, Korkos DP, et al. Dual PI3K/mTOR Inhibition in Colorectal Cancers with APC and PIK3CA Mutations. Mol Cancer Res. 2017;15(3):31727. doi: 10.1158/1541-7786.mcr-16-0256.

72. Jung JW, Veitch M, Bridge JA, Overgaard NH, Cruz JL, Linedale R, et al. Clinically-Relevant Rapamycin Treatment Regimens Enhance CD8(+) Effector Memory T Cell Function In The Skin and Allow their Infiltration into Cutaneous Squamous Cell Carcinoma. Oncoimmunology. 2018;7(9):e1479627. doi: 10.1080/2162402x.2018.1479627.

73. Hirayama Y, Gi M, Yamano S, Tachibana H, Okuno T, Tamada S, et al. Anti-PD-L1 treatment enhances antitumor effect of everolimus in a mouse model of renal cell carcinoma. Cancer Sci. 2016;107(12):1736-44. doi: 10.1111/cas.13099.

74. Wang Y, Song F, Zhu J, Zhang S, Yang Y, Chen T, et al. GSA: Genome Sequence Archive<sup/>. Genomics Proteomics Bioinformatics. 2017;15(1):14-8. doi: 10.1016/j.gpb.2017.01.001.

75. Database Resources of the National Genomics Data Center, China National Center for Bioinformation in 2021. Nucleic Acids Res. 2021;49(D1):D18-d28. doi: 10.1093/nar/gkaa1022.

\section{Figures}

\section{Figure 1}

Expression of MTOR in CRC.(a) The expression of MTOR in the majority of human cancers differed from normal tissues. ${ }^{\star} p<0.05,{ }^{* \star} p<0.01,{ }^{* \star *} p<0.001$. The blue boxes represent normal tissues; the red boxes represent tumor tissues; the purple boxes represent metastatic tumor tissues. The box plots display MTOR expression levels in CRC and corresponding normal tissues from TCGA (b) and the CRC cohorts GSE41657 (c) and GSE113513 (d). * $p<0.05$. (e) Representative immunohistochemistry (IHC) staining showing differences in $\mathrm{mTOR}$ expression between CRC and paired normal tissues. (f) The box plot displays the IHC scores for mTOR in CRC and corresponding normal tissues from the Ruijin cohort. CRC, colorectal cancer; TCGA, The Cancer Genome Atlas; mTOR, mammalian target of rapamycin. 


\section{Figure 2}

Prognostic value of MTOR in CRC patients. ( $\mathrm{a}$ and $\mathrm{b}$ ) Kaplan-Meier survival curve comparing high ( $\mathrm{N}=$ $279)$ and low $(\mathrm{N}=280)$ MTOR expression determined by the median value and high ( $\mathrm{N}=140)$ and low ( $\mathrm{N}$ = 140) MTOR expression determined by quantile value for the TCGA CRC patient cohort. (c and d) Kaplan-Meier survival curve comparing high $(\mathrm{N}=95)$ and low $(\mathrm{N}=96)$ MTOR expression determined by the median value and high $(\mathrm{N}=47)$ and low $(\mathrm{N}=47)$ MTOR expression determined by quantile value for the CRC patients in the GSE87211 database. CRC, colorectal cancer; TGCA, The Cancer Genome Atlas.

\section{Figure 3}

Identification of DEGs.(a and b) The heatmap and volcano plot show differentially expressed genes (DEGs) in CRC patients with different MTOR expression levels; "down" indicates downregulated DEGs, and "up" indicates upregulated DEGs; "stable" indicates that the difference was not significant. (c) The top 15 pathways analyzed by KEGG were mapped according to DEGs. (d) GO showed the Top 15 of BP, CC, and MF (BP, Biological Process; CC, Cellular Component; MF, Molecular Function). The GSEA results showed that the terms "ECM-receptor interaction" (e), "focal adhesion" (f), "regulation of actin cytoskeleton" (g), "adherens junction" (h), "ribosome" (i), "oxidative phosphorylation" (j), "fatty acid metabolism" (k), and "glutathione metabolism" (I) were differentially enriched between the different MTOR expression levelsin CRC patients. CRC, colorectal cancer; KEGG, Kyoto Encyclopedia of Genes and Genomes; GSEA, gene set enrichment analysis.

\section{Figure 4}

Mutation signatures in CRC.(a) Summary of the mutation information among $537 \mathrm{CRC}$ patients from TCGA. Classification of mutation types according to different categories; tumor mutation burden in specific patients; the top 10 mutated genes in CRC. (b) The oncoplot displays the somatic landscape of the CRC cohort. Genes are ordered by their mutation frequency. Sidebar plot shows $\log _{10}$-transformed Qvalues estimated by MutSigCV. (c) The oncoplot shows the frequency of mutations in genes associated with the mTOR pathway. CRC, colorectal cancer; TGCA, The Cancer Genome Atlas, mTOR, mammalian target of rapamycin.

\section{Figure 5}


Association between MTOR and mutation signatures in CRC. Significantly mutated genes associated with low (a) and high (b)MTOR expression levels in CRC patients. The oncoplot shows the different somatic landscapes of CRC patientsbetween the low (c) and high (d)MTOR expression groups. Individual patients are presented in each column. (e) Differentially mutated genes between the high and low MTOR expression groups are displayed as the forest plot. Bars indicate the $95 \%$ confidence interval for the odds ratio. The adjacent table includes the number of patients in the high and low MTOR expression groups with the mutations in the highlighted gene. ${ }^{* *} p<0.001$. (f) The boxplot shows differences in the tumor mutational burden between high and low MTOR expression groups. CRC, colorectal cancer.

\section{Figure 6}

Correlation analysis between MTOR expression and tumor-infiltrating immune cells (TIICs) in CRC.(a) The scatterplots display the purity-corrected Spearman's rho values and $p$-values for the correlations between MTOR and TIICs. (b) The boxplot showed the correlation between MTOR copy number variation and TIICs in CRC. ${ }^{*} p<0.05, * \star * p<0.001$. (c) The stacked bar chart shows the different TIIC proportions between high and low MTOR expression groups. (d) The box plot shows TIIC fraction differences between high and low MTOR expression groups. ns, not significant, ${ }^{*} \mathrm{p}<0.05$, ${ }^{*} \mathrm{p}<0.01,{ }^{* \star} \mathrm{p}<<0.001$. (e) The box plot shows expression differences among immune checkpoint genes between high and low MTOR expression groups. ${ }^{\star} p<0.05,{ }^{\star \star} p<0.01,{ }^{\star \star \star} p<0.001$. CRC, colorectal cancer.

\section{Figure 7}

Correlation analysis between MTOR expression in CRC and MSI.(a) The boxplot shows the different MTOR expression levels between MSI-H and MSS CRC patients from GSE75316. (b) Representative IHC staining shows differences in mTOR expression between MSI and MSS CRC tissues. (c) Thewestern blots show protein expression levels of mTOR and p-mTOR in CRC cell lines. The MSI cell lines RKO and HCT116 and the MSS cell lines HT-29 and SW-620 are shown. CRC, colorectal cancer; MSI, microsatellite instability; MSI-H, MSI-high; MSS; microsatellite stable; mTOR, mammalian target of rapamycin.Fulllength blots are presented in Supplementary Fig.3-5.

\section{Figure 8}

Mutation signatures of MTOR in CRC with MSI. Characteristics of the variations identified in MTOR in MSI-H (a) and MSS (b) CRC patients. The boxplots show the differences in variant classifications (c), variant types (d), SNV classifications (e), transitions, and transversion (f) among MTOR mutations in MSI 
and MSS CRC patients. ns, not significant, ${ }^{*} p<0.05,{ }^{*} p<0.01$. CRC, colorectal cancer; MSI, microsatellite instability; MSI-H, MSI-high; MSS; microsatellite stable; SNV, single-nucleotide variant.

\section{Figure 9}

MTOR mutation in sub-groups of clinical characteristics. MTOR mutation frequency analyses were stratified based on MSI status (a), age (b), tumor location (c), vascular invasion (d), T stage (e), M stage (f), N Stage (g), and TMN stage (h). p-value $<0.05$ was used to assess differences. MSI, microsatellite instability; T, tumor; $M$, metastasis; $N$, node.

\section{Supplementary Files}

This is a list of supplementary files associated with this preprint. Click to download.

- Supplementaryfig.3.jpg

- Supplementaryfig.4.jpg

- Supplementaryfig.5.jpg

- supplementaryfig.1.jpg

- supplementaryfig.2.jpg 